\title{
The use of simulation training module to train surgical residents in the focused assessment with sonography for trauma (FAST)
}

\author{
Shetty, $P .^{1}$, Sherif, $L .^{2} \&$ Mijar, $M .^{3}$
}

\begin{abstract}
Introduction: The focused assessment with sonography for trauma (FAST) is a commonly used and lifesaving tool in the initial assessment of trauma patients. Surgical residents being trained through lectures and hands-on learning of ultrasound can be challenging; inclusion of novel educational strategies, such as simulation can be utilized. Thus this study ought to evaluate whether ultrasound simulator is a suitable alternative method for ultrasound education than the traditional methods.
\end{abstract}

Method: Observational analytical study. Sixteen surgical residents were taken for the study, by lottery chit system they were segregated into to arm of eight each. One arm was with traditional hands on training and another arm was taught in Simulation skill lab on interpretation of FAST. The duration of study was 6 weeks, 2 hours per week. Knowledge content of FAST evaluated trough Pre-test and Post MCQ test (Identical) and OSCE assessment was done for their skill and interpretation of FAST. Evaluator was blinded to both groups. Chi square and student $t$ test was used for statistical analysis.

Result: All students showed significant improvement from pre- to post-test scores and significant improvement in comfort level using ultrasound in general and on FAST $(p<0.001)$. There was no significant difference between groups on OSCE scores. Overall, no differences were demonstrated between groups trained on human models versus simulator.

Conclusion: These findings suggest that an ultrasound simulator is a suitable alternative method for ultrasound education

Keywords: Trauma, Blunt Abdomen, Hemoperitoneum, FAST, Simulation, Innovative teaching

\section{Introduction}

A substantial number of patients presenting to emergency medicine have blunt abdominal injury and it is of utmost importance to diagnose hemoperitoneum quickly.

${ }^{1}$ Associate professor, Department of General surgery, Father Muller Medical College and Hospital, Karnataka

${ }^{2}$ Academic In charge, Father Muller Simulation and Skill Centre, Karnataka, India

${ }^{3}$ Assistant Professor, Department of Radio diagnosis and Imaging, Father Muller Medical College and Hospital, Karnataka, India

Corresponding Author:

Dr. Prathvi Shetty

Associate professor, Department of General surgery, Father Muller Medical College and Hospital, Father Muller Road, Kankanady, Mangalore- 575002, Karnataka, India

Email:prathviz@fathermuller.in
During resuscitation of blunt abdominal trauma patients, FAST is often the first diagnostic imaging modality for patient evaluation. It is especially invaluable for those who are hemodynamically unstable, to rapidly screen for any free fluid (Hsu et al., 2007; Patel \& Riherd, 2011). The early detection of free fluid in peritoneum, pericardium and pleural space becomes of utmost importance to first responders who usually tend to be surgical residents in our set up.

FAST skills are considered essential for physicians and are not difficult to learn and practice. The accuracy of FAST performed by non-radiologists has been well documented in the published literature (Brenchley et al., 2006; Mazur et al., 2008). Most of the training happens using the traditional teaching on human patients which are often unstructured,

DOI: http://doi.org/10.4038/seajme.v13i1.58

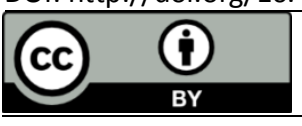

14

(C) SEAJME. This is an Open Access article distributed under the terms of the Creative Commons Attribution

License (http://creativecommons.org/licenses/by/4.0/), which permits unrestricted use, distribution, and reproduction in any medium, provided the original author and source are credited

Vol. 13, no. 1, 2019


expensive and time-consuming, requiring the use of live human models, instructors and ultrasound machines (Ahern et al., 2010). The use of simulators as educational tools for medical procedures is spreading rapidly. Recent studies of usage of ultrasound simulators have shown, that the confidence of using sonography and in image interpretation skills there were no difference in groups who have been trained using patient or those who have been trained using simulators (Bentley et al., 2015; Damewood et al., 2011; Barsuk et al., 2012). The aim of this study was to compare simulator-based ultrasound training module to the traditional method of using human models.

\section{Methodology}

\section{Study Design}

This was a parallel, blinded, randomized controlled study conducted on a convenient sample of first and third year surgical residents totaling to 16 in number. This study was approved by the institutional ethical board, participation was voluntary and written consent was obtained from all participants.

\section{Study Setting and Population}

This study was done over six weeks with two hours dedicated every week. No formal ultrasound education exists in the surgical resident curriculum at our institution. Students were randomized into a traditional training group (control group) or an ultrasound simulator group (intervention group). Randomization was done with unbiased independent observer lottery chit method. It included eight in control group and eight in intervention group, each group had an equal mix of first and third year surgical residents.

\section{Study Protocol}

Prior to the training, all students took a 15question written pre-test, composed of questions from The American Institute of Ultrasound in Medicine (AIUM) AIUM Practice Parameter for the Performance of the Focused Assessment With Sonography for Trauma (FAST) Examination prepared in conjunction with the American College of Emergency Physicians (ACEP), targeting emergency department ultrasound indications, contraindication and equipment specifications (Bahner et al., 2008). All students received a standardized, introductory lecture on the use of ultrasound, FAST basics and indications and how to conduct a FAST exam. The lecture was delivered by the same instructor to both control and intervention groups.

The control group participated in hands-on learning and were trained by radiology teaching staff and the intervention group participated in hands-on learning and practice of the FAST exam on the CAE Vimedix ultrasound simulator which is a torso model with embedded electronics that simulates high fidelity normal and pathologic images in real time as the students perform ultrasound scans. Following the training modules, all students completed a post-test (identical to the pre-test). Additionally, an objective structured clinical examination (OSCE) was administered to both groups (Sisley et al., 1999). Outcomes based on the following measures were evaluated: comparison of ultrasound knowledge between pre and post-test scores in order to assess ultrasound knowledge; and finally the results of the OSCE scores were compared between groups. Students in OSCE were assessed on their performance of the FAST exam which include the usage of ultrasound technique, the ability to effectively visualize each ultrasound view, image acquisition time and diagnosing pathological video clips. The assessor was blinded to both groups.

\section{Statistical analysis}

Categorical variables were expressed by frequency and percentage. In order to confirm the improved level of knowledge and skill before and after the training, the test results underwent unpaired and paired sample t-test. The statistical analysis was performed using SPSS version 18 (IBM Inc., Chicago, USA) and $p$ value under 0.05 was considered statistically significant.

\section{Result}

A total of 16 students were trained and tested in this study (control group $\mathrm{N}=8$, intervention group $\mathrm{N}=8$ ). The pre-test scores, on indication, contraindication, equipment specifications survey and basic FAST exam showed no significant difference between both groups. Mean pre-test and post test scores for the control group were 8.5 (SD 2.32) and 11.75 (SD 1.66) respectively. Mean pre-test and post-test scores for the intervention group were 8.5 (SD 2.32) and 11.75 (SD 1.38) (Table 1). Comparison of scores between groups showed no significant difference, though there was a significant improvement in their pre- and posttest scores $(p<0.001)$, understandably because 
of intervention leading to increase in the clinical knowledge.

There was no significant difference between groups on the OSCE standardized, clinical skills checklist. Mean OSCE score was 4.25 for the control group (C) and 4.88 for the intervention group (I) (Table 2). The window acquisition time (Table 3) for Right Upper
Quadrant (C- 2.4 mins \& I- 2.3 mins), Left Upper Quadrant (C- 2.5 mins \& I- 3.4 mins), Pelvic View (C- 1.3 mins \& I- 1.4 mins), Sub Xiphoid View (C- 2.1 mins \& I- 1.5mins), Total scan (C5.6 mins \& I- 6.2 mins). Between both groups there was no appreciable or statistically significant difference in any of the described variables.

Table 1: Mean pre-test and post test scores for the control (C) and Intervention (I) group

\begin{tabular}{lcccc}
\hline & \multicolumn{2}{c}{ Pre Test } & \multicolumn{2}{c}{ Post Test } \\
\cline { 2 - 5 } & Mean & $\begin{array}{c}\text { Standard } \\
\text { Deviation (SD) }\end{array}$ & Mean & $\begin{array}{c}\text { Standard } \\
\text { Deviation (SD) }\end{array}$ \\
\hline Control (C) & 8.5 & 2.32 & 11.75 & 1.66 \\
Intervention (I) & 8.5 & 2.32 & 11.75 & 1.38 \\
\hline \multicolumn{5}{c}{ P value $=0.00316$} \\
\hline
\end{tabular}

Table 2: Mean OSCE score for the control group (C) and Intervention (I) group

\begin{tabular}{lcccc}
\hline & Mean & $\begin{array}{c}\text { Standard } \\
\text { Deviation (SD) }\end{array}$ & $\begin{array}{c}\text { Confidence } \\
\text { Interval (Cl) 95\% }\end{array}$ & $\begin{array}{c}\text { Standard Error } \\
\text { (SE) }\end{array}$ \\
\hline Control (C) & 4.25 & 0.99 & 1.960 & $3.43-4.81$ \\
Intervention (I) & 4.88 & 0.353 & 1.960 & $4.63-5.12$ \\
\hline \multicolumn{5}{r}{} \\
\end{tabular}

Table 3: The window acquisition time

\begin{tabular}{lcc}
\hline & Control (C) & Intervention(I) \\
\hline Right Upper Quadrant (RUQ) & 2.4 & 2.3 \\
Left Upper Quadrant (LUQ) & 2.5 & 3.4 \\
SX & 2.1 & 1.5 \\
Pelvic & 1.3 & 1.4 \\
Total & 5.6 & 6.2 \\
\hline
\end{tabular}




\section{Discussion}

The purpose of FAST training are for the trainee to identify free intraperitoneal fluid accurately as well as analysis of the pericardium and pleural spaces for haemorrhage.

In our study it was hypothesized that using an ultrasound simulator for basic ultrasound training for the FAST would not be less effective to traditional teaching on human model. All residents showed an increase in ultrasound knowledge after the educational intervention. The intervention group also exhibited similar scores in OSCE, which represents skills gains that is window acquisition, window quality, ultrasound technique and time to scan. Traditional teaching using human models involves direct faculty time, a dedicated ultrasound machine and to attain proficiency by performing the FAST exam on as many individuals, or cases, as possible with varying pathology is impractical and time consuming.

The use of an ultrasound simulator streamlines the educational process by obviating the need for human patients and additional ultrasound machines for training purposes (Brenchley et al., 2006). The simulators which we had used provided real-time imaging and visual feedback of the anatomy and also with various pathological scenarios. Thus allowing the trainee to be exposed to far more cases than would have been in real life scenarios. This gives the trainee more time to practice and thereby increasing proficiency and skill level, thus adhering to what principles ACEP advocates (American College of Emergency Physicians, 2001). Another notable advantage was, it provide learning opportunities to practice skills in a controlled, safe environment which reduces stress for the student and potential harm to patient (Gibbs, 2015). As it is selfdirected learning, students could come in their convenient time to train.

\section{Limitations}

The study include a small sample size $(\mathrm{N}=16)$. The primary aim of simulated learning should be to prepare the student who has acquired the necessary knowledge and skill for practice in the real clinical setting but here the translatability of these skills to clinical scenarios was not addressed.

\section{Conclusion}

The use of an ultrasound simulator is a convenient and objective method of training surgical residents on ultrasound allowing standardization of education. The main advantage of simulator over traditional training is time and repeat practice which can be manipulated to meet the needs of the student and tailored to their learning pace in a risk-free environment. Simulation can be used to train surgical resident although there are questions as to its value in terms of the overall clinical experience.

\section{References}

Ahern, M., Mallin, M.P., Weitzel, S., Madsen, T. \& Hunt, P. (2010) Variability in ultrasound education among emergency medicine residencies, Western Journal of Emergency Medicine, 11, 4, p. 314

American College of Emergency Physicians, (2001) American College of Emergency Physicians, ACEP emergency ultrasound guidelines-2001, Annals of emergency medicine, 38, 4, p. 470

Bahner, D., Blaivas, M., Cohen, H.L., Fox, J.C., Hoffenberg, S., Kendall, J., Langer, J., McGahan, J.P., Sierzenski, P. \& Tayal, V.S. (2008) AIUM practice guideline for the performance of the focused assessment with sonography for trauma (FAST) examination, Journal of ultrasound in medicine: official journal of the American Institute of Ultrasound in Medicine, 27, 2, pp. 313-318

Barsuk, J.H., Cohen, E.R., Vozenilek, J.A., O'Connor, L.M., McGaghie, W.C. \& Wayne, D.B. (2012) Simulation-based education with mastery learning improves paracentesis skills, Journal of graduate medical education, 4, 1, pp. 23-27

Bentley, S., Mudan, G., Strother, C. \& Wong, N. (2015) Are live ultrasound models replaceable? Traditional versus simulated education module for FAST exam, Western Journal of Emergency Medicine, 16, 6, p. 818

Brenchley, J., Walker, A., Sloan, J.P., Hassan, T.B. \& Venables, H. (2006) Evaluation of focussed assessment with sonography in trauma (FAST) by UK emergency physicians, Emergency Medicine Journal, 23, 6, pp. 446-448

Damewood, S., Jeanmonod, D. \& Cadigan, B. (2011) Comparison of a multimedia simulator to a human model for teaching FAST exam image interpretation and image acquisition, Academic Emergency Medicine, 18, 4, pp. 413-419 
Gibbs, V. (2015) The role of ultrasound simulators in education: an investigation into sonography student experiences and clinical mentor perceptions, Ultrasound, 23, 4, pp. 204-211

Hsu, J.M., Joseph, A.P., Tarlinton, L.J., Macken, L. \& Blome, S. (2007) The accuracy of focused assessment with sonography in trauma (FAST) in blunt trauma patients: experience of an Australian major trauma service, Injury, 38, 1, pp. $71-75$

Mazur, S.M., Pearce, A., Alfred, S., Goudie, A. \& Sharley, P. (2008) The FASTER trial: focused assessment by sonography in trauma during emergency retrieval: a feasibility study, Injury, 39,5 , pp. $512-518$
Patel, N.Y. \& Riherd, J.M. (2011) Focused assessment with sonography for trauma: methods, accuracy, and indications, The Surgical clinics of North America, 91, 1, pp. 195207

Sisley, A.C., Johnson, S.B., Erickson, W. \& Fortune, J.B. (1999) Use of an Objective Structured Clinical Examination (OSCE) for the assessment of physician performance in the ultrasound evaluation of trauma, Journal of Trauma and Acute Care Surgery, 47, 4, p. 627 